\title{
AN UPPER BOUND ON THE NUMBER OF PARITY CHECKS FOR BURST ERROR DETECTION AND CORRECTION IN EUCLIDEAN CODES
}

\author{
SAPna Jain And Ki-Suk LeE
}

\begin{abstract}
There are three standard weight functions on a linear code viz. Hamming weight, Lee weight, and Euclidean weight. Euclidean weight function is useful in connection with the lattice constructions [2] where the minimum norm of vectors in the lattice is related to the minimum Euclidean weight of the code. In this paper, we obtain an upper bound over the number of parity check digits for Euclidean weight codes detecting and correcting burst errors.
\end{abstract}

\section{Introduction}

There are three standard weight functions (or equivalently distance/metric functions) on a linear code viz. Hamming weight $[1,4,14]$, Lee weight $[7,10$, $11,12,13]$ and Euclidean weight $[2,6]$. The choice of a metric for a given communication system plays an important role as the channel model should match the metric $d$ to be employed for developing a suitable code, and hence for a communication system to operate reliably. Thus given a modulation scheme, one metric may be better suited than another. Euclidean weight (or Euclidean square distance) is useful in connection with the latice construction where the minimum norm of vectors in the lattice is related to the minimum Euclidean weight of the code [2]. Also, it is well known that during the process of transmission errors occur predominantly in the from of bursts. However, it does not generally happen that all the digits inside any burst length gets corrupted. In other words, the weight of the burst errors are not large. Codes developed to detect and correct burst errors with respect to Hamming and Lee weight functions have been studied by many authors [3, 5, 10, 12] and [15]. In this paper, we first obtain an upper bound for Euclidean codes detecting burst errors and then we obtain an upper bound for codes correcting such type of errors.

In what follows, we consider the following:

Received November 19, 2007.

2000 Mathematics Subject Classification. 94B05.

Key words and phrases. Euclidean weight, linear codes, minimum distance, burst errors.

(C)2009 The Korean Mathematical Society 
Let $\mathbb{Z}_{q}$ be the ring of integers modulo $q$. Let $V_{q}^{n}$ be the set of all $n$-(tuples) over $\mathbb{Z}_{q}$. Then $V_{q}^{n}$ is a module over $\mathbb{Z}_{q}$. Let $V$ be a submodule of the module $V_{q}^{n}$ over $\mathbb{Z}_{q}$. For $q$ prime, $\mathbb{Z}_{q}$ becomes a field and correspondingly $V_{q}^{n}$ and $V$ become the vector space and subspace respectively over the field $\mathbb{Z}_{q}$. Also, we define the Euclidean value $|a|^{2}$ of an element $a \in \mathbb{Z}_{q}$ by

$$
|a|^{2}= \begin{cases}a^{2} & \text { if } \quad 0 \leq a \leq q / 2, \\ (q-a)^{2} & \text { if } \quad q / 2<a \leq q-1,\end{cases}
$$

or in other words

$$
|a|^{2}=\min \left(a^{2},(q-a)^{2}\right),
$$

then, for a given vector $u=\left(a_{0}, a_{1}, \ldots, a_{n-1}\right), a_{i} \in \mathbb{Z}_{q}$, the Euclidean weight $w_{E}(u)$ of $u$ is given by

$$
w_{E}(u)=\sum_{i=0}^{n-1}\left|a_{i}\right|^{2} .
$$

Note that in determining the Euclidean weight of vector, a nonzero entry $a$ has a contribution $|a|^{2}$ which is obtained by two different entries $a$ and $q-a$ provided $\{q$ is odd $\}$ or $\{q$ is even and $a \neq q / 2\}$. That is,

$$
|a|^{2}=|q-a|^{2} \quad \text { if }\left\{\begin{array}{l}
q \text { is odd } \\
\text { or } \\
q \text { is even and } a \neq q / 2 .
\end{array}\right.
$$

If $q$ is even and $a=q / 2$ or if $a=0$, then $|a|^{2}$ is obtained in only one way viz. $|a|^{2}=a^{2}$.

Thus for the Euclidean weight, there may be one or two entries from $\mathbb{Z}_{q}$ having the same Euclidean value $|a|^{2}$ and we call these entries as repetitive equivalent Euclidean values of $a$. The number of repetitive equivalent Euclidean values of $a$ will be denoted by $e_{a}$, where

$$
e_{a}= \begin{cases}1 & \text { if }\{q \text { is even and } a=q / 2\} \text { or }\{a=0\} \\ 2 & \text { if }\{q \text { is odd and } a \neq 0\} \text { or }\{q \text { is even, } a \neq 0 \text { and } a \neq q / 2\} .\end{cases}
$$

The Euclidean square distance between the two vectors $u=\left(a_{0}, a_{1}, \ldots, a_{n-1}\right)$ and $v=\left(b_{0}, b_{1}, \ldots, b_{n-1}\right)$ is defined as the Euclidean weight of their difference, i.e.,

$$
d_{E}^{2}(u, v)=w_{E}(u-v) .
$$

The minimum Euclidean square distance of a code is the smallest Euclidean square distance between all its distinct pair of code words. Also, minimum Euclidean square distance $\left(d_{E}^{2}\right)$ and minimum Euclidean weight of a code coincide.

We note that Euclidean weight (or equivalent Euclidean square distance) of a vector over $\mathbb{Z}_{q}$ can assume values which can be expressed as sum of squares of positive integers and the integers are chosen from the set $\{0,1,2,3, \ldots,[q / 2]\}$.

We shall denote $[x]$ as the largest integer less than or equal to $x$. 


\section{An upper bound for Euclidean weight codes detecting burst errors}

In this section, we obtain an upper bound on the number of parity check digits for codes detecting burst errors of length $b$ or less with Euclidean weight $w_{E}$ or less $\left(1 \leq w_{E} \leq b[q / 2]^{2}\right)$.

To prove the result, we first prove the following lemma:

Lemma 2.1. If $V_{t}^{(n)}$ denotes the number of all $n$-tuples of Euclidean weight $t$ or less over $\mathbb{Z}_{q}$, then $V_{t}^{(n)}$ is given by

$$
V_{t}^{(n)}=\sum_{\eta=0}^{t} A_{\eta}^{(n)}
$$

where

(2) $A_{\eta}^{(n)}= \begin{cases}\sum_{N=1}^{[q / 2]} \sum_{\substack{r_{0}, r_{1} \ldots, r_{N} \\ 1}} \frac{n !}{r_{0} ! r_{1} ! \cdots r_{N} !} e_{1}^{r_{1}} e_{2}^{r_{2}} \cdots e_{N}^{r_{N}} & \text { for } \eta>0 \\ & \text { for } \eta=0,\end{cases}$

and $r_{i}^{\prime} s$ are integers such that

$$
\begin{aligned}
& r_{0}+r_{1}+r_{2}+\cdots+r_{N}=n, \quad r_{N} \geq 1, \quad r_{i} \geq 0 \quad(i \neq N), \\
& 1^{2} r_{1}+2^{2} r_{2}+\cdots+N^{2} r_{N}=\eta .
\end{aligned}
$$

Proof. Clearly, $A_{\eta}^{(n)}=1$ for $\eta=0$ is obvious. So, assume $\eta \geq 1$. We consider partitions of the integer $\eta(1 \leq \eta \leq t)$, the largest entry in which has an equivalent Euclidean value $N^{2}(1 \leq N \leq[q / 2])$. If $r_{i}$ is the number of times, entries with equivalent Euclidean value $|i|^{2}$ occurs in the partition, then the number of vectors of length $n$ that can be formed by filling $n$ positions from the integers $0,1, \ldots, N$ is given by

$$
\frac{n !}{r_{0} ! r_{1} ! \cdots r_{N} !} e_{1}^{r_{1}} e_{2}^{r_{2}} \cdots e_{N}^{r_{N}}
$$

Condition (3) follows immediately as the total number of entries is $n$ and the sum of Euclidean values of the entries is $\eta$. Now summing (4) for all possible values of $r_{i}^{\prime} s$ and of $N(1 \leq N \leq[q / 2])$, we get (2). Finally, $V_{t}^{(n)}$ is obtained by summing $A_{\eta}^{(n)}$ for all possible values of $\eta$ which range from 0 to $t$. Hence the proof is completed.

We now give a definition:

Definition 2.1. A linear combination of $n$-vectors $u_{1}, u_{2}, \ldots, u_{n}$ given by

$$
\alpha_{1} u_{1}+\alpha_{2} u_{2}+\cdots+\alpha_{n} u_{n},
$$

where $\alpha_{i} \in \mathbb{Z}_{q}, u_{i} \in \mathbb{Z}_{q}^{n}(1 \leq i \leq n)$ is called a linear combination of Euclidean weight $w_{E}$ if

$$
\text { Euclidean weight }\left(\alpha_{1}, \alpha_{2}, \ldots, \alpha_{n}\right)=w_{E} \text {. }
$$


Theorem 2.1. Given positive integers $b$ and $w_{E}\left(1 \leq w_{E} \leq b[q / 2]^{2}\right)$, a sufficient condition that there exists an $(n, k)$ linear code over $\mathbb{Z}_{q}$ ( $q$ prime) that has no burst of length $b$ or less with Euclidean weight $w_{E}$ or less as a code word is

$$
q^{n-k}>1+\sum_{l=1}^{[q / 2]} e_{l} V_{w_{E}-l^{2}}^{(b-1)}
$$

Proof. The existence of such a code will be proved by constructing a suitable $(n-k) \times n$ parity check matrix $H$ for the desired code. We select any non zero $(n-k)$-tuple as the first column of $H$. Subsequent columns are added to $H$ in such a way that after having selected $j-1$ columns $h_{1}, h_{2}, \ldots, h_{j-1}$ suitably, a nonzero $(n-k)$-tuple is chosen as the $j^{\text {th }}$ column $h_{j}$ such that

$$
\lambda h_{j} \neq \lambda_{i_{1}} h_{i_{1}}+\lambda_{i_{2}} h_{i_{2}}+\cdots+\lambda_{i_{p}} h_{i_{p}},
$$

where

$$
\lambda, \lambda_{i_{j}} \in \mathbb{Z}_{q}(j=1 \text { to } p),\left|\lambda_{i_{1}}\right|^{2}+\left|\lambda_{i_{2}}\right|^{2}+\cdots+\left|\lambda_{i_{p}}\right|^{2}+|\lambda|^{2} \leq w_{E}
$$

and

$$
\left\{h_{i_{1}}, h_{i_{2}}, \ldots, h_{i_{p}}\right\} \subseteq\left\{h_{j-1}, h_{j-2}, \ldots, h_{j-b+1}\right\},
$$

i.e., $h_{j}$ is chosen in such a way that no linear combination of Euclidean weight $w_{E}$ or less from the immediately preceding $b-1$ columns and column $h_{j}$ is zero i.e., no linear combination of Euclidean weight $w_{E}$ or less from the columns $h_{j-b+1}, h_{j-b+2}, \ldots, h_{j-1}, h_{j}$ is zero. Such a condition will ensure that a burst of length $b$ or less with Euclidean weight $w_{E}$ or less cannot be a code vector in the code whose parity check matrix is $H$.

The number of possible linear combinations in equation (6) including the pattern of all zeros is given by

$$
1+e_{1} V_{w_{E}-1^{2}}^{(b-1)}+e_{2} V_{w_{E}-2^{2}}^{(b-1)}+\cdots+e_{[q / 2]} V_{w_{E}-[q / 2]^{2}}^{(b-1)}=1+\sum_{l=1}^{[q / 2]} e_{l} V_{w_{E}-l^{2}}^{(b-1)}
$$

Therefore, a column $h_{j}$ can be added to $H$ provided that this number is less than the total number of $(n-k)$-tuples which is $q^{n-k}$.

At worst, all these linear combinations might yield a distant sum, therefore, $h_{j}$ can always be added to $H$ provided that

$$
q^{n-k}>1+\sum_{l=1}^{[q / 2]} e_{l} V_{w_{E}-l^{2}}^{(b-1)}
$$

It is important to note that the relation in equation (7) is independent of $j$. Therefore, we can go on adding the columns as long as we wish but for code of length $j$, we shall stop after choosing $j$ columns. So for $j=n$, we shall add up to $n$ columns.

This completes the proof of the theorem. 
Example 2.1. Consider the following $3 \times 4$ parity check matrix of a $(4,1)$ linear Euclidean weight code over $\mathbb{Z}_{5}$ :

$$
H=\left[\begin{array}{llll}
1 & 0 & 0 & 2 \\
0 & 1 & 0 & 2 \\
0 & 0 & 1 & 3
\end{array}\right]_{3 \times 4}
$$

This matrix has been constructed by the synthesis procedure outlined in the proof of Theorem 2.1 by taking $b=3$ and $w_{E}=4$. The code whose parity check matrix is $H$ satisfies the sufficient condition of Theorem 2.1 as shown below:

$$
\begin{aligned}
1+\sum_{l=1}^{[q / 2]} e_{l} V_{w_{E}-l^{2}}^{(b-1)}= & 1+\sum_{l=1}^{2} e_{l} V_{4-l^{2}}^{(2)} \\
= & 1+e_{1} V_{3}^{(2)}+e_{2} V_{0}^{(2)} \\
= & 1+(2 \times 9)+(2 \times 1) \\
& \left(\text { since } V_{3}^{(2)}=9 \text { and } V_{0}^{(2)}=1\right) \\
= & 21 .
\end{aligned}
$$

Also, $q^{n-k}=5^{3}=125$.

Therefore,

$$
q^{n-k}=125>21=1+\sum_{l=1}^{[q / 2]} e_{l} V_{w_{E}-l^{2}}^{(b-1)} .
$$

By Theorem 2.1 we get that the code detects all burst errors of length 3 or less with Euclidean weight 4 or less. We verify this fact as follows:

The generator matrix $G$ of the code corresponding to the above given parity check matrix $H$ is given by

$$
G=\left[\begin{array}{llll}
3 & 3 & 2 & 1
\end{array}\right]_{1 \times 4}
$$

The code words of this code are

$$
\begin{gathered}
v_{0}=0000, \text { Euclidean weight }\left(v_{0}\right)=0 \\
v_{1}=3321, \text { Euclidean weight }\left(v_{1}\right)=13 \\
v_{2}=1142, \text { Euclidean weight }\left(v_{2}\right)=7 \\
v_{3}=4413, \text { Euclidean weight }\left(v_{3}\right)=7 \\
v_{4}=2234, \text { Euclidean weight }\left(v_{4}\right)=13 .
\end{gathered}
$$

Thus, all the code words of the code whose parity check matrix is $H$ are not bursts of length 3 or less with Euclidean weight 4 or less over $\mathbb{Z}_{5}$, i.e., code detects all bursts of length 3 or less with Euclidean weight 4 or less over $\mathbb{Z}_{5}$. 


\section{An upper bound for Euclidean weight codes correcting burst errors}

In this section, we obtain an upper bound on the number of parity check digits for codes correcting burst errors of length $b$ or less with Euclidean weight $w_{E}$ or less $\left(1 \leq w_{E} \leq b[q / 2]^{2}\right)$.

Theorem 3.1. A sufficient condition for the existence of an $(n, k)$ linear Euclidean weight code over $\mathbb{Z}_{q}$ ( $q$ prime) which corrects all bursts of length $b$ or less $(n>2 b)$ with Euclidean weight $w_{E}$ or less $\left(1 \leq w_{E} \leq b[q / 2]^{2}\right)$ is given by

(8)

$$
\begin{aligned}
q^{n-k}> & +\left[\sum_{\lambda=1}^{[q / 2]} e_{\lambda} V_{w_{E}-\lambda^{2}}^{(b-1)}\right]\left[\sum_{i=1}^{b}(n-b-i+1)\left(V_{w_{E}}^{(i)}-1\right)\right] \\
& +\sum_{\lambda=1}^{[q / 2]} e_{\lambda}\left(V_{2 w_{E}-\lambda^{2}}^{(b-1)}-V_{1-\lambda^{2}}^{(b-1)}\right) \\
& +\sum_{\lambda=1}^{[q / 2]} e_{\lambda}\left(\sum_{k=1}^{(b-1)} \sum_{\lambda_{1}=1}^{\min \left\{[q / 2],\left[\sqrt{w_{E}-1}\right]\right\}} \sum_{r_{\lambda_{1}}, r_{2 \lambda_{1}}, r_{3 \lambda_{1}}} e_{\lambda_{1}} A_{r_{\lambda_{1}}}^{(b-k-1)} A_{r_{2} \lambda_{1}}^{(k)} A_{r_{3 \lambda_{1}}}^{(b-k-1)}\right),
\end{aligned}
$$

where

$$
\begin{aligned}
2-\lambda^{2} & \leq \lambda_{1}^{2}+r_{1 \lambda_{1}}+r_{2 \lambda_{1}}+r_{3 \lambda_{1}} \leq 2 w_{E}-\lambda^{2}, \\
1 & \leq \lambda_{1}^{2}+r_{1 \lambda_{1}} \leq w_{E}-1, \\
1 & \leq r_{2 \lambda_{1}} \leq 2 w_{E}-1-\lambda^{2}, \\
0 & \leq r_{3 \lambda_{1}} \leq w_{E}-\lambda^{2}, \\
r_{2 \lambda_{1}} & +r_{3 \lambda_{1}} \geq 1-\lambda^{2}, \\
\lambda_{1}^{2} & +r_{1 \lambda_{1}}+r_{2 \lambda_{1}} \geq 1 .
\end{aligned}
$$

Proof. The existence of such a code will be proved by constructing a suitable $(n-k) \times n$ parity check matrix $H$ for the desired code. We select any nonzero $(n-k)$-tuple as the first column of parity check matrix $H$. Subsequent columns are added to $H$ in such a way that after having selected $j-1$ columns $h_{1}, h_{2}, \ldots, h_{j-1}$ suitably, a nonzero $(n-k)$-tuple is chosen as the $j^{\text {th }}$ column $h_{j}$ such that $\alpha h_{j}(1 \leq \alpha \leq q-1)$ is not a linear combination of any number of columns of Euclidean weight $w_{E}-|\alpha|^{2}$ or less from the immediately preceding $b-1$ columns $h_{j-b+1}, h_{j-b+2}, \ldots, h_{j-1}$ together with any number of columns with Euclidean weight $w_{E}$ or less among any $b$ consecutive columns out of all the $j-1$ columns selected so far. In other words, column $h_{j}$ can be added to $H$ provided that

(10) $\alpha h_{j} \neq\left(\alpha_{i_{1}} h_{i_{1}}+\alpha_{i_{2}} h_{i_{2}}+\cdots+\alpha_{i_{r}} h_{i_{r}}\right)+\left(\beta_{j_{1}} h_{j_{1}}+\beta_{j_{2}} h_{j_{2}}+\cdots+\beta_{j_{m}} h_{j_{m}}\right)$, where $1 \leq \alpha \leq q-1$ (or, equivalently $1 \leq|\alpha|^{2} \leq[q / 2]^{2}$ ), $\alpha_{i_{1}} h_{i_{1}}+\alpha_{i_{2}} h_{i_{2}}+$ $\cdots+\alpha_{i_{r}} h_{i_{r}}$ is any linear combination of Euclidean weight less than or equal to 
$w_{E}-|\alpha|^{2}$ of the columns from $h_{j-b+1}, h_{j-b+2}, \ldots, h_{j-1}$ and $\beta_{j_{1}} h_{j_{1}}+\beta_{j_{2}} h_{j_{2}}+$ $\cdots+\beta_{j_{m}} h_{j_{m}}$ is any linear combination of Euclidean weight $w_{E}$ or less from a set of $b$ consecutive columns among all $j-1$ columns.

To compute the number of all possible linear combinations occurring in (10) for all possible choices of $\alpha_{i_{j}}$ 's and $\beta_{j_{k}}$ 's, we analyze the situation in three different cases.

Case 1. When $\beta_{j_{k}}$ 's in Equation (10) are taken from the first $j-b$ columns. It is clear that

$$
1 \leq \sum_{k=1}^{m}\left|\beta_{j_{k}}\right|^{2} \leq w_{E}
$$

and

$$
0 \leq \sum_{j=1}^{r}\left|\alpha_{i_{j}}\right|^{2} \leq w_{E}-|\alpha|^{2},
$$

where $1 \leq|\alpha|^{2} \leq[q / 2]^{2}$.

The number of $\beta_{j_{k}}$ 's satisfying equation (11) is

$$
\sum_{i=1}^{b}(j-b-i+1)\left(V_{w_{E}}^{(i)}-V_{0}^{(i)}\right)=\sum_{i=1}^{b}(j-b-i+1)\left(V_{w_{E}}^{(i)}-1\right) .
$$

The number of $\alpha_{i_{j}}$ 's satisfying inequality (12) is

$$
\sum_{\lambda=1}^{[q / 2]} e_{\lambda} V_{w_{E}-\lambda^{2}}^{(b-1)}
$$

Case 2. When $\beta_{j_{k}}$ 's are taken from the immediately preceding $b-1$ columns.

In this case, the number of linear combinations occurring in equation (10) or, in other words, number of additional ways in which $\alpha_{i_{j}}$ 's and $\beta_{j_{k}}$ 's can be selected is given by

$$
\sum_{\lambda=1}^{[q / 2]} e_{\lambda}\left(V_{2 w_{E}-\lambda^{2}}^{(b-1)}-V_{1-\lambda^{2}}^{(b-1)}\right)
$$

Case 3. When $\beta_{j_{k}}$ 's are neither completely confined to the first $j-b$ columns nor to the last $b-1$ columns.

In this case, $h_{j_{k}}$ 's are selected from $h_{j-2 b+2}, h_{j-2 b+3}, \ldots, h_{j-1}$ in such a way that not all are taken either from $h_{j-2 b+2}, h_{j-2 b+3}, \ldots, h_{j-b}$ or from $h_{j-b+1}$, $h_{j-b+2}, \ldots, h_{j-1}$. Let us suppose that the burst starts from the $(j-2 b+k+1)^{t h}$ position which may continue upto $(j-b+k)^{t h}$ position $(1 \leq k \leq b-1)$. Let the Euclidean value of the element occurring at the starting position $(j-$ $2 b+k+1)$ of the burst be $\lambda_{1}^{2}$. Further, let us have linear combinations, of Euclidean weight $r_{1 \lambda_{1}}$, of columns from the $(j-2 b+k+1)^{t h}, \ldots,(j-b)^{t h}$ columns; linear combinations of Euclidean weight $r_{2 \lambda_{1}}$, of columns from the $(j-b+1)^{t h}, \ldots,(j-b+k)^{t h}$ columns; and linear combinations of Euclidean 
weight $r_{3 \lambda_{1}}$, of columns from the $(j-b+k+1)^{t h}, \ldots,(j-1)^{t h}$ columns. Then, in this case, the total number of choices of the linear combinations of equation (10) turns out to be

(16) $\sum_{\lambda=1}^{[q / 2]} e_{\lambda}\left(\sum_{k=1}^{(b-1)} \sum_{\lambda_{1}=1}^{\min \left\{[q / 2],\left[\sqrt{w_{E}-1}\right]\right\}} \sum_{r_{1 \lambda_{1}}, r_{2 \lambda_{1}}, r_{3 \lambda_{1}}} e_{\lambda_{1}} A_{r_{1 \lambda_{1}}}^{(b-k-1)} A_{r_{2 \lambda_{1}}}^{(k)} A_{r_{3 \lambda_{1}}}^{(b-k-1)}\right)$,

where

$$
\begin{aligned}
2-\lambda^{2} & \leq \lambda_{1}^{2}+r_{1 \lambda_{1}}+r_{2 \lambda_{1}}+r_{3 \lambda_{1}} \leq 2 w_{E}-\lambda^{2}, \\
1 & \leq \lambda_{1}^{2}+r_{1 \lambda_{1}} \leq w_{E}-1, \\
1 & \leq r_{2 \lambda_{1}} \leq 2 w_{E}-1-\lambda^{2}, \\
0 & \leq r_{3 \lambda_{1}} \leq w_{E}-\lambda^{2}, \\
r_{2 \lambda_{1}}+r_{3 \lambda_{1}} & \geq 1-\lambda^{2}, \\
\lambda_{1}^{2}+r_{1 \lambda_{1}}+r_{2 \lambda_{1}} & \geq 1 .
\end{aligned}
$$

Thus total number of possible distinct linear combinations arising out of all the three cases including the patterns of all zeros is given by

$$
\begin{aligned}
& 1+\left[\sum_{\lambda=1}^{[q / 2]} e_{\lambda} V_{w_{E}-\lambda^{2}}^{(b-1)}\right]\left[\sum_{i=1}^{b}(j-b-i+1)\left(V_{w_{E}}^{(i)}-1\right)\right]+\sum_{\lambda=1}^{[q / 2]} e_{\lambda}\left(V_{2 w_{E}-\lambda^{2}}^{(b-1)}-V_{1-\lambda^{2}}^{(b-1)}\right) \\
& +\sum_{\lambda=1}^{[q / 2]} e_{\lambda}\left(\sum_{k=1}^{(b-1)} \sum_{\lambda_{1}=1}^{\min \left\{[q / 2],\left[\sqrt{w_{E}-1}\right]\right\}} \sum_{r_{\lambda_{1}}, r_{2 \lambda_{1}}, r_{3 \lambda_{1}}} e_{\lambda_{1}} A_{r_{1 \lambda_{1}}}^{(b-k-1)} A_{r_{2 \lambda_{1}}}^{(k)} A_{r_{3 \lambda_{1}}}^{(b-k-1)}\right)=: L,
\end{aligned}
$$

where $r_{1 \lambda_{1}}, r_{2 \lambda_{1}}, r_{3 \lambda_{1}}$ satisfy the constraints given in inequalities (17).

Therefore, a column $h_{j}$ can be added to the parity check matrix $H$ provided that

$$
q^{n-k}>L
$$

But for an $(n, k)$ linear code to exist, the inequality (18) should hold for $j=n$ and we get $(8)$.

This completes the proof of the theorem.

Example 3.1. Take $b=2, w_{E}=2, q=5, n=5, k=1$. We show the existence of a $(5,1)$ linear code over $\mathbb{Z}_{5}$ satisfying the sufficient condition (8) and correcting all burst errors of length 2 or less with Euclidean weight 2 or less.

We now compute the value of R.H.S. of inequality (8) for above mentioned values of parameters, i.e., for $b=2, w_{E}=2, q=5, n=5, k=1$.

$$
\begin{aligned}
(\text { R.H.S. }) \text { of }(8)= & +\sum_{\lambda=1}^{2} e_{\lambda}\left[V_{2-\lambda^{2}}^{(1)}\left(3\left(V_{2}^{(1)}-1\right)+2\left(V_{2}^{(2)}-1\right)\right)\right. \\
& +\left(V_{4-\lambda^{2}}^{(1)}-V_{1-\lambda^{2}}^{(1)}\right)
\end{aligned}
$$




$$
\begin{aligned}
& \left.+\sum_{k=1}^{1} \sum_{\lambda_{1}=1}^{1} \sum_{r_{\lambda_{1}}, r_{\lambda_{1}}, r_{3 \lambda_{1}}} e_{\lambda_{1}} A_{r_{\lambda_{\lambda_{1}}}}^{(1-k)} A_{r_{\lambda_{1}}}^{(k)} A_{r_{3 \lambda_{1}}}^{(1-k)}\right] \\
= & 1+\sum_{\lambda=1}^{2} e_{\lambda} M_{\lambda}
\end{aligned}
$$

where

$$
\begin{aligned}
M_{\lambda}= & V_{2-\lambda^{2}}^{(1)}\left(3\left(V_{2}^{(1)}-1\right)+2\left(V_{2}^{(2)}-1\right)\right)+\left(V_{4-\lambda^{2}}^{(1)}-V_{1-\lambda^{2}}^{(1)}\right) \\
& +\sum_{k=1}^{1} \sum_{\lambda_{1}=1}^{1} \sum_{r_{1 \lambda_{1}}, r_{2 \lambda_{1}}, r_{3 \lambda_{1}}} e_{\lambda_{1}} A_{r_{1 \lambda_{1}}}^{(1-k)} A_{r_{2 \lambda_{1}}}^{(k)} A_{r_{3 \lambda_{1}}}^{(1-k)},
\end{aligned}
$$

and

$$
\begin{aligned}
& 2-\lambda^{2} \leq \lambda_{1}^{2}+r_{1 \lambda_{1}}+r_{2 \lambda_{1}}+r_{3 \lambda_{1}} \leq 4-\lambda^{2}, \\
& 1 \leq \lambda_{1}^{2}+r_{1 \lambda_{1}} \leq 1 \\
& 1 \leq r_{2 \lambda_{1}} \leq 3-\lambda^{2} \\
& 0 \leq r_{3 \lambda_{1}} \leq 2-\lambda^{2} \\
& r_{2 \lambda_{1}}+r_{3 \lambda_{1}} \geq 1-\lambda^{2}, \\
& \lambda_{1}^{2}+r_{1 \lambda_{1}}+r_{2 \lambda_{1}} \geq 1 .
\end{aligned}
$$

Since $\lambda$ varies from 1 to 2 in equation (19), therefore, we compute $M_{\lambda}$ corresponding to each value of $\lambda$ in the following two cases:

Case 1. When $\lambda=1$.

In this case, $r_{1 \lambda_{1}}, r_{2 \lambda_{1}}, r_{3 \lambda_{1}}$ have following three sets of feasible solutions:

(i) $r_{1 \lambda_{1}}=0, \quad r_{2 \lambda_{1}}=1, \quad r_{3 \lambda_{1}}=1$

(ii) $r_{1 \lambda_{1}}=0, \quad r_{2 \lambda_{1}}=1, \quad r_{3 \lambda_{1}}=0$

(iii) $r_{1 \lambda_{1}}=0, \quad r_{2 \lambda_{1}}=2, \quad r_{3 \lambda_{1}}=0$.

Therefore, the value of $M_{\lambda}$ in (19) for $\lambda=1$ is given by

$$
\begin{aligned}
\left.M_{\lambda}\right|_{\lambda=1}= & V_{1}^{(1)}\left(3\left(V_{2}^{(1)}-1\right)+2\left(V_{2}^{(2)}-1\right)\right)+\left(V_{3}^{(1)}-V_{0}^{(1)}\right) \\
& +2\left(A_{0}^{(0)} A_{1}^{(1)} A_{1}^{(0)}+A_{0}^{(0)} A_{1}^{(1)} A_{0}^{(0)}+A_{0}^{(0)} A_{2}^{(1)} A_{0}^{(0)}\right) \\
= & 3(3 \times 2+2 \times 8)+(3-1)+2(0+0+0) \\
= & 68 .
\end{aligned}
$$

Case 2. When $\lambda=2$.

For this case, there is no feasible solution for $r_{1 \lambda_{1}}, r_{2 \lambda_{1}}, r_{3 \lambda_{1}}$. Therefore, the value of $M_{\lambda}$ in (19) for $\lambda=2$ is taken to be zero, i.e.,

$$
\left.M_{\lambda}\right|_{\lambda=2}=0
$$


Substituting the values of $M_{\lambda}$ for $\lambda=1,2$ from (20) and (21) respectively in equation (19), we get

$$
\begin{aligned}
(19) & =1+e_{1} \times 68+e_{2} \times 0 \\
& =1+2 \times 68+2 \times 0 \\
& =137 .
\end{aligned}
$$

Also, L.H.S. of $(8)$ for a $(5,1)$ linear code over $\mathbb{Z}_{5}=5^{n-k}=5^{4}=625$.

Since $625>137$, therefore, the sufficient condition $(8)$ is satisfied for a $(5,1)$ linear code over $\mathbb{Z}_{5}$ for $b=2, w_{E}=2$.

Now, consider the following $4 \times 5$ parity check matrix of a $(5,1)$ linear code over $\mathbb{Z}_{5}$ :

$$
H=\left[\begin{array}{lllll}
1 & 0 & 0 & 0 & 3 \\
0 & 1 & 0 & 0 & 0 \\
0 & 0 & 1 & 0 & 2 \\
0 & 0 & 0 & 1 & 1
\end{array}\right]_{4 \times 5}
$$

This matrix has been constructed by the synthesis procedure outlined in the proof of Theorem 3.1 by taking $b=2$ and $w_{E}=2$. The null space of this matrix is the desired code which corrects all burst errors of length 2 or less with Euclidean weight 2 or less. It can be seen from the following table that syndromes of all the correctable error patterns, i.e., all burst errors of length 2 or less with Euclidean weight 2 or less are distinct.

Table.

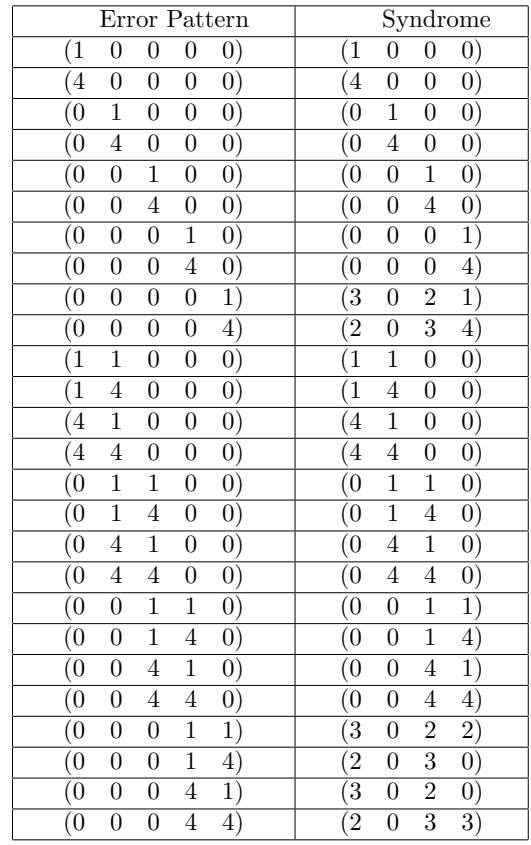


Acknowledgment. The first author would like to thank her husband Arihant Jain for his constant inspiration and encouragement for pursuing research.

\section{References}

[1] E. R. Berlekamp, Algebraic Coding Theory, McGraw-Hill Book Co., New York-Toronto, Ont.-London 1968.

[2] A. Bonnecaze, P. Solé, and A. R. Calderbank, Quaternary quadratic residue codes and unimodular lattices, IEEE Trans. Inform. Theory 41 (1995), no. 2, 366-377.

[3] C. N. Campopiano, Bounds on Burst Error Correcting Codes, IRE. Trans. IT-8 (1962), 257-259.

[4] W. Cary Huffman and V. Pless, Fundamentals of Error-Correcting Codes, Cambridge University Press, 2003.

[5] P. Fire, A Class of Multiple-Error-Correcting Binary Codes for Non-Independent Errors, Sylvania Reports RSL-E-2, Sylvania Reconnaissance Systems, Mountain View, California, 1959.

[6] S. Jain, Error detecting and error correcting capabilities of Euclidean codes, Pragmatic algebra, 51-66, SAS Int. Publ., Delhi, 2006.

[7] __ Modification to a bound for random error correction with Lee weight, Commun. Korean Math. Soc. 20 (2005), no. 2, 405-409.

[8] S. Jain, On a sufficient condition to attain minimum square distance in Euclidean codes, to appear in Algebra Colloquium.

[9] S. Jain and S. H. Choi, Plotkin's bound in codes equipped with Euclidean weight function, to appear in Tamsui Oxford Journal of Mathematics.

[10] S. Jain and K.-B. Nam, Lower bounds for codes correcting moderate-density bursts of fixed length with Lee weight consideration, Linear Algebra Appl. 418 (2006), no. 1, $122-129$.

[11] S. Jain, K.-B. Nam, and K.-S. Lee, On some perfect codes with respect to Lee metric, Linear Algebra Appl. 405 (2005), 104-120.

[12] S. Jain and K. P. Shum, Sufficient condition over the number of parity checks for burst error detection/correction in linear Lee weight codes, Algebra Colloq. 14 (2007), no. 2, $341-350$.

[13] C. Y. Lee, Some properties of non-binary error correcting codes, IEEE Trans. Information Theory IT-4 (1958), 77-82.

[14] W. W. Peterson and E. J. Weldon, Jr., Error Correcting Codes, 2nd Edition, MIT Press, Cambridge, Massachusetts, 1972.

[15] A. D. Wyner, Low-density-burst-correcting Codes, IEEE. Trans. Information Theory IT-9 (1963), 124.

SAPNA JAIN

Department of Mathematics

UNIVERSITY OF DELhi

DELHI 110 007, INDIA

E-mail address: sapna@vsnl.com

KI-SUK LEE

Department of Mathematics Education

Korea National University of Education

Cheongwon 363-791, Korea

E-mail address: kslee@knue.ac.kr 